\title{
Newly Seen Debris Disks from the HST NICMOS Archive
}

\author{
Marshall D. Perrin ${ }^{1}$, Elodie Choquet ${ }^{1}$, Christine Chen $^{1}$, John Debes ${ }^{1}$, \\ David Golimowski ${ }^{1}$, J. Brendan Hagan ${ }^{1}$, Dean C. Hines ${ }^{1}$, \\ Tushar Mittal $^{1,2}$, Margaret Moerchen ${ }^{1}$, Mamadou N'Diaye ${ }^{1}$, \\ Laurent Pueyo $^{1,2}$, I. Neill Reid ${ }^{1}$, Glenn Schneider ${ }^{3}$, Schuyler Wolff ${ }^{2}$ \\ and Remi Soummer ${ }^{1}$ \\ ${ }^{1}$ Space Telescope Science Institute, 3700 San Martin Dr, Baltimore, MD, 21218 USA \\ ${ }^{2}$ Johns Hopkins University, Baltimore MD, 21218 USA \\ ${ }^{3}$ University of Arizona, Tucson AZ, 85721 USA
}

\begin{abstract}
By reprocessing the NICMOS coronagraphic archive using improved PSF subtraction methods, we have obtained new images of 5 debris disks, all previously unseen using classical PSF subtractions. Three of the disks are edge on and two appear to be ring like, one of which is extremely asymmetric.

Their stellar hosts are nearby, young $\mathrm{F}$ and $\mathrm{G}$ type stars (40-90 pc, 12-30 Myr), including one that is a close analog to the young sun at roughly the age at which terrestrial planets were assembling. This is a $25 \%$ increase in the sample of debris disks seen in scattered light. Analysis and modeling of the disk geometries is in process. Given these systems' youth, proximity, and brightness $(\mathrm{V}=7.2$ to 8.5$)$, these will be superb targets for investigating planet formation, and are perfect targets for studies with GPI, SPHERE and JWST.
\end{abstract}

Keywords. circumstellar matter, planetary systems, techniques: image processing

\section{Background}

About 20 debris disks have previously been seen in scattered light, and a roughly similar number resolved in thermal emission. These imaged disks represent only a tiny portion of the thousands of nearby disks whose existence can be inferred from infrared excesses. But these imaged disks are tremendously valuable for advancing our understanding of nearby planetary systems. Due to the inherent degeneracies of SED-only modeling, imaging observations are essential to unambiguously determine the locations of debris and parent body belts and to ascertain the properties of the light-scattering dust grains.

\section{Methods}

By reprocessing the NICMOS coronagraphic archive using improved PSF subtraction methods, we have obtained new images of 5 debris disks, all previously unseen using classical PSF subtractions. The key elements of our reprocessing include:

(a) Careful recalibration and removal of NICMOS instrumental artifacts using optimal reference files for darks, flats, etc. ("LAPLACE" HST AR Program; PI Schneider)

(b) PSF subtraction using the KLIP algorithm (Soummer, Pueyo \& Larkin 2012) applied to a large library of reference PSFs drawn from contemporaneous science programs. See the article on the ALICE pipeline by Choquet et al. in these proceedings.

(c) Disk images from multiple rolls were then merged using the iterative roll subtraction algorithm of Krist et al. 2010 to further reduce time-variable residuals. 

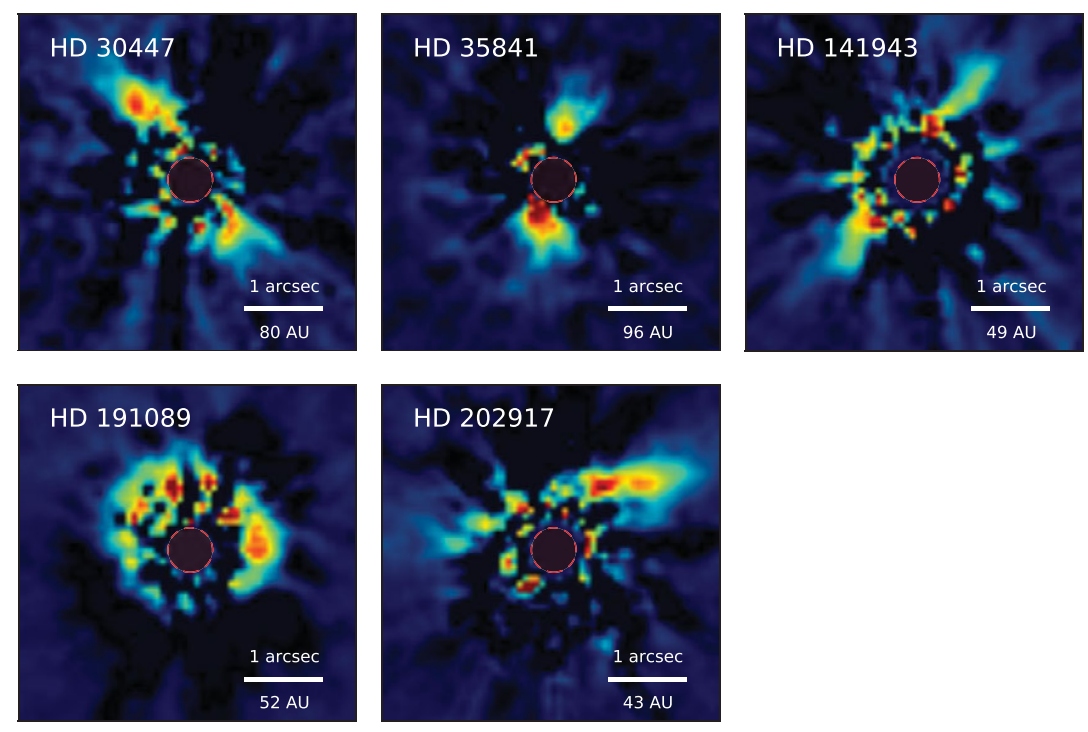

Figure 1. HST NICMOS F110W images of five debris disks, all on identical angular scale and oriented to have north up. These images show the combination of 2 rolls for each target; the disks are also seen consistently in the individual rolls but at lower $\mathrm{S} / \mathrm{N}$. Two of these disks have prior imaging detections at other wavelengths (HD 191089 at $18 \mu \mathrm{m}$, Churcher et al. 2011; HD 202917 in unpublished ACS GTO observations, Krist 2007) and in those cases properties such as disk position angles and inclinations are broadly consistent.

Compared to the prior existing sample of HST-imaged disks, these newly seen disks are preferentially young ( $<30 \mathrm{Myr})$ and near solar type (G7-F2). This is the result of selection biases in the NICMOS surveys from which we drew the data, which targeted young nearby systems for planet and disk searches.

\section{Results}

See Figure 1. HD 30447, a F3V star at 80 pc and member of the Columba Association, is seen to have a roughly edge-on disk, about $50 \%$ brighter on its northeast side. HD 38541, another F3V member of Columba, has the faintest and most compact disk in our sample, detected at $r<1.5^{\prime \prime}$. HD 141943, a G2V star at $49 \mathrm{pc}$, is the first solar-type star $<50 \mathrm{Myr}$ in age to have a debris disk imaged in scattered light. It has the lowest IR excess in our sample, $L_{I R} / L_{\star} \sim 1.2 \mathrm{e}-4$. An edge on disk is seen from $0.7-2.6^{\prime \prime}=$ 35-125 AU in projected separation. HD 191089, an F5V star at 52 pc and member of the $\beta$ Pic moving group, shows an intermediate inclination $\left(i \sim 30^{\circ}\right)$ disk with one side significantly brighter than the other. HD 202917, a G7V star at 43 pc and member of the Tucana-Horologium association, shows a striking and dramatic asymmetric arc, suggestive of a partial ring seen near $i \sim 70^{\circ}$, which is precisely aligned with the similar one-sided "needle" detected in prior ACS GTO observations (Krist et al. 2007).

Acknowledgements: Support for HST AR 12652 was provided by NASA through a grant from STScI, which is operated by AURA under NASA contract NAS5-26555.

\section{References}

Churcher, L., Wyatt, M., \& Smith, R. 2011, MNRAS, 410, 2

Krist, J. E., Stapelfeldt, K. R., Bryden, G., et al. 2010, AJ, 140, 1051

Krist, J. 2007, Proceedings of the "Spirit of Lyot" conference.

Soummer, R., Pueyo, L. \& Larkin, J. 2012, ApJ, 755, L28 